

\title{
8 mortalidad en la niñez en 16 municipios del corredor turístico centro-sur de Honduras
}

\author{
GLAdYs Elizabeth MenJívar
}

El estado de salud de una población es considerado como una de las más fieles expresiones de su bienestar general, éste a su vez está condicionado por la operación de factores determinantes más generales tales como la acción de salud pública, el avance de las tecnologías médicas, el desarrollo económico, la calidad del medio ambiente, el progreso social y las condiciones políticas imperantes en una nación. Es por ello que la mortalidad, como resultado final de los diferentes estados específicos de enfermedad que afectan a la población, es por su parte un indicador no sólo de cómo actúan los factores antes mencionados, sino también la expresión final de la acción única o combinada de ciertos determinantes próximos, los que en ultima instancia, se encargan de filtrar la influencia de tales factores y modelan la estructura final de las causas de muerte que actúan en cada contexto (Albizu-Campos. J; 1990).

Es así cómo la información sobre las condiciones de la mortalidad de una población es frecuentemente considerada como la fuente de indicadores confiables del bienestar, aun cuando ella, por si sola, no contribuya de forma significativa a la explicación de la acción de los mecanismos causales que le dan lugar a este fenómeno social, en tanto ella es sólo la forma visible y transfigurada de un proceso más complejo y acumulativo de trastornos biológicos que pueden tentativamente finalizar en una defunción -que es lo que en última instancia se registra con mayor o menor calidad-y que es conducido por una combinación de condicionantes sociales y médicas.

Todos sabemos la importancia que tiene el tema de la mortalidad en niños/as de 1-5 años, en una población. (La mortalidad de 0-5 años incluye: la mortalidad infantil, mortalidad en la niñez).Con frecuencia se utiliza el nivel de mortalidad infantil como uno de los indicadores, para clasificar a los países de acuerdo al nivel socioeconómico alcanzado. (Welti, Carlos, 1997. Pág. 88).

\section{Objetivos de la investigación:}

\section{Objetivo General}

Analizarlos diferenciales de la mortalidad en la niñez, en 16 municipios del corredor turístico centro sur de Honduras.

\section{Objetivos específicos:}

-Analizar los diferenciales de la mortalidad en la niñez en 16 municipios del corredor turístico centro- sur de Honduras según condiciones de la vivienda.

- Analizar los diferenciales de la mortalidad en la niñez en 16 municipios del corredor turístico centro- sur de Honduras según educación de la madre.

- Analizar los diferenciales de la mortalidad en la niñez según área de residencia.

\section{Hipótesis:}

- La mortalidad en la niñez en los 16 municipios del corredor turístico centro- sur de Honduras es mayor en el área rural que en área urbana.

- La mortalidad en la niñez en los 16 municipios del corredor turístico centro- sur de Honduras es menor a mayor educación de la madre.

- La mortalidad en la niñez en los 16 municipios del corredor turístico centro- sur de Honduras es menor, si son mejores las condiciones de la vivienda referidas a tipo de piso, existencia de servicio sanitario, abastecimiento adecuado de agua y forma de eliminación de la basura.

MORTALIDAD A NIVEL MUNDIAL, AMERICA LATINA Y HONDURAS

El Informe sobre el Estado Mundial de la Infancia menciona que para el año 2000 las estimaciones sobre mortalidad infantil registran promedios que van desde 163 muertes por mil nacidos vivos en Sierra Leona, 138 en Guinea Bissau, 146 en Mozambique (todos países de África); en comparación con Italia, España, Eslovaquia 7 por mil nacidos vivos, Japón 4 muertes por mil nacidos vivos.

En el caso de América Latina según el boletín № 62 de CELADE, Haití tenía en el período 70- 75 tasas de 152.2, Honduras 103.7, Cuba el más bajo de todos 38.5 muertes por mil nacidos vivos; para el período 1995-2000, Haití 66 muertes por mil nacidos vivos el más alto, Honduras 35.0, Cuba 9 el más bajo de todos los países de América Latina más alto Haití, Honduras, en el medio y en mejor situación Cuba.

En el caso de Centro América la tendencia al descenso se mantiene, obsérvese tabla.

Algunos autores se preguntan porque ha bajado la tasa de mortalidad infantil aun en condiciones de deterioro de las economías.

La discusión en este sentido es si el descenso de la mortalidad, especialmente la infantil ha sido el resultado de desarrollo socioeconómico que han ido experimentando los países en desarrollo o bien por la aplicación de programas explícitos en materia de salud y de la importación de tecnología medica y sanitaria. La discusión ha planteado ambas explicaciones como alternativas excluyentes y la preponderancia de uno u otro factor (condiciones de vida o programas de salud) es todavía objeto de debate.

Otros autores (Mosley y Chen) proponen "un modelo para el estudio de la supervivencia en la infancia en países en desarrollo que incorpore tanto la perspectiva de las ciencias sociales como de las ciencias medicas". 
Tasas de Mortalidad Infantil en Centro América Período 19802000

(Por cada mil nacidos vivos)

$\begin{array}{lrrrrr}\text { Países } & \mathbf{1 9 8 0} & \mathbf{1 9 8 5} & \mathbf{1 9 9 0} & \mathbf{1 9 9 5} & \mathbf{2 0 0 0} \\ \text { Guatemala } & 82.4 & 78.8 & 65 & 51.1 & 46.0 \\ \text { El Salvador } & 87.3 & 77.0 & 54.0 & 40.2 & 32.0 \\ \text { Honduras } & 81.0 & 65.0 & 53.0 & 43.0 & 35.0 \\ \text { Nicaragua } & 96.6 & 79.8 & 65.0 & 48.1 & 43.4 \\ \text { Costa Rica } & 30.4 & 19.2 & 16.0 & 13.7 & 12.1 \\ \text { Panamá } & 35.4 & 30.4 & 28.4 & 25.1 & 21.4\end{array}$

Fuente: CEPAL (Comisión Económica para América Latina y el Caribe) Boletín Demográfico №66, Santiago de Chile, 2000

La presente investigación se realizó en 16 municipios del llamado Corredor Turístico Centro- Sur, cuyo criterio de selección fue la ubicación de estos municipios respecto a la Carretera Panamericana, con el propósito de conocer cual era el potencial turístico y las condiciones de vida de sus pobladores, por lo tanto, no podía dejarse de estudiar la mortalidad en la niñez, pues este es considerado como un indicador clásico de las condiciones de vida de una población.

\section{METODOLOGÍA}

Generalmente las fuentes de información que se utilizan para el estudio de la mortalidad infantil y en la niñez son las estadísticas vitales, los censos nacionales de población y las encuestas de salud.

Las estadísticas vitales se refieren a la información continua sobre hechos vitales como por ejemplo nacimientos, defunciones, matrimonios que generalmente es recopilada por el registro civil. En Honduras el organismo recolector de estos datos es el registro nacional de las personas y procesada por el INE.

Teóricamente, las estadísticas vitales deberían ser la fuente principal que proporcione la información necesaria para estimar la mortalidad general, e infantil de una población, desgraciadamente en Honduras, las estadísticas vitales son muy deficientes , para estudiar a través de ellas la mortalidad en la niñez, uno de los problemas principales tiene relación con el subregistro, es decir la carencia del registro del hecho vital, sucede que si un niño nace hoy y muere mañana, este no fue registrado ni como nacido vivo ni como muerto, y este panorama se agudiza más sobre la causa de muerte y es probable que la omisión sea mayor en aquellas causas relacionadas con enfermedades infecciosas y parasitarias. Por lo tanto el estudio de la mortalidad es doblemente limitado y esta situación no solo sucede en Honduras si no en la mayoría de los países en vías de desarrollo.

Es por esta situación que se han desarrollado procedimientos alternativos o técnicas indirectas para estimar la mortalidad infantil, estos se basan en información recopilada a través de preguntas especiales sobre el tema ya sea en un censo de población y vivienda o en una encuesta demográfica de salud.

Para el desarrollo de este trabajo se utilizó el procedimiento ideado por W Brass, variante Coale- Trussell, tomando como fuente de información los datos que sobre mortalidad infantil y juvenil se recopilaron en el último censo de población y vivienda realizado en el país en el año 2001.

La información utilizada se refiere concretamente, sobre la pregunta que aparece en el censo sobre hijos nacidos vivos e hijos sobrevivientes, clasificados por edad de la madre, esta información se clasifica de acuerdo a cada variable seleccionada : área de residen- cia, nivel educativo de la madre y condiciones de la vivienda en los siguientes municipios: El Triunfo, Namasigue, Marcovia, Choluteca, San Antonio de Flores y Pespire en el departamento de Choluteca; Santa Lucia, Ojojona, Valle de Ángeles, Santa Ana, La Venta, San Buenaventura, Sabanagrande en el departamento de Francisco Morazán; Goascoran, San Lorenzo y Nacaome en el departamento de Valle. Utilizando para tal efecto el paquete computacional Redatam en donde se realizaron los cruces de variables, y el Pandem en donde se procesaron los datos.

MÉTODO DE ESTIMACIÓN DE LA MORTALIDAD INFANTIL Y JUVENIL A PARTIR DE LA INFORMACIÓN SOBRE HIJOS NACIDOS VIVOS E HIJOS SOBREVIVIENTES CLASIFICADOS POR EDAD DE LA MADRE.

Como ya explicaba anteriormente debido a los problemas de calidad que presentan las estadísticas vitales, se han desarrollado procedimientos alternativos para el estudio de la mortalidad, en este caso explicare el ideado por W Brass, esta metodología requiere como información básica la siguiente:

- Población femenina clasificada por grupos quinquenales de edad, de 15 a 49 años (en el caso de mujeres que son madres en las edades comprendidas de 10-14 años se sumaron al grupo de $15-19$ años y en el caso de mujeres que son madres de los 50 años en adelante se suman a los del grupo de 45- 49 años para efectos del estudio.

- Número de hijos nacidos vivos, clasificados por grupos quinquenales de edad de las madres.

- Número de hijos sobrevivientes o fallecidos enumerados según grupo de edad de las madres.

Esta información se recopila al momento de realizar el censo, para el caso en el censo del 2001 se pregunta.

Para mujeres mayores de 12 años

¿Cuántos hijos nacidos vivos ha tenido?

De estos nacidos vivos

¿Cuántos han fallecido?

¿Cuántos están actualmente vivos?

Con estos datos es posible entonces calcular la proporción de hijos fallecidos respecto al total de hijos nacidos vivos según edad de la madre.

En cuanto al análisis de los resultados hay que considerar que los q (x) obtenidos no pueden considerarse como valores exactos: por ejemplo los q (1) es uno de los menos confiables ya que al depender de las mujeres muy Jóvenes (15-19) puede no solo verse afectado por omisiones o errores, sino también por el hecho de que la mortalidad en la niñez de los hijos nacidos de mujeres muy jóvenes no es representativo, así mismo se ha verificado en muchos casos que la mortalidad derivada de los grupos de mujeres de edades mayores por encima de los 40 años puede estar afectada por la omisión de declaración de hijos nacidos vivos e hijos sobrevivientes.

Los comentarios anteriores dejan en evidencia que los resultados obtenidos en la aplicación del método deben interpretarse con cierta prudencia.

También es importante aclarar que el método indirecto ideado por Brass, no se recomienda se utilice en el estudio de pequeñas poblaciones, por que cuando se utiliza los datos pueden presentar alguna inestabilidad.

En definitiva cualquiera que sea el método que se utilice son los datos básicos recogidos sobre el terreno, sobre el total de hijos tenidos 
y sobrevivientes los que determinaran con mayor o menor validez la bondad de las estimaciones que se obtengan.

\section{DISCUSION Y RESULTADOS}

A continuación se presentan los resultados obtenidos a través de la aplicación el método indirecto para ello se utilizó la base de datos de los 16 municipios ubicados en el corredor turístico centrosur de Honduras.

Como bien la aclara Brass el método no se recomienda para el estudio de pequeñas poblaciones, entonces lo que se hizo fue agrupar los municipios en se respectivo departamento.

En Choluteca: Marcovia, Namasigue, El Triunfo, Pespire, Choluteca, San Antonio de Flores.

En Francisco Morazán: Santa Ana, Santa Lucia, Valle de Ángeles, Sabanagrande, La Venta, Ojojona, San Buenaventura.

En Valle: Nacaome, Goascoran y San Lorenzo.

La población que fue objeto de estudio fue la que se muestra en la tabla No. 1.

Tomando en cuenta esa población se analizó los resultados de las diferencias de mortalidad en la niñez según el área de residencia, el nivel educativo de la madre y las condiciones de saneamiento de la vivienda.

Es importante señalar que para el análisis de la mortalidad en la niñez se tomaron los valores encontrados en q (2) de 20-24 años y q (3) de 25 - 29 años de los resultados obtenidos en el programa Pandem, pues como lo explica Brass y se menciona en la metodología los q (1) y q (7) no son confiables para el análisis de la mortalidad por las razones aclaradas anteriormente.

También se debe recalcar que cuando se desagrega la información por distintos niveles: departamento, municipios, áreas de residencia, el caso nuestro municipios con pequeñas poblaciones, la información puede presentar problemas de inestabilidad debido al reducido número de observaciones en algunas categorías de análisis.

\section{VARIABLE AREA DE RESIDENCIA}

Se espera que las tasas de mortalidad en la niñez sean mayores en el área rural que en el área urbana.

Tabla No. 1. Población Objeto de Estudio

\begin{tabular}{lcc} 
Departamento & $\begin{array}{c}\text { Población objeto } \\
\text { de estudio }\end{array}$ & $\begin{array}{c}\text { Población total del } \\
\text { departamento }\end{array}$ \\
\hline Francisco Morazán & 59,506 & 1180,676 \\
Choluteca & 269.863 & 390.815 \\
Valle & 59,506 & 151,841
\end{tabular}

Fuente: Elaboración propia a través de método de Brass, en base a los datos de XVI censo de población y vivienda, INE, Honduras, 2001.

Tabla No. 2. Tasas de la mortalidad en la niñez estimada a partir del método de Brass variante Coale- Trussell (modelo oeste) por área de residencia urbana rural.

\begin{tabular}{lll}
\multicolumn{1}{c}{ Departamento } & Área urbana & Área rural \\
\hline Choluteca & 24 & 33 \\
Francisco Morazán & 18 & 29 \\
Valle & 17 & 26
\end{tabular}

Fuente: Elaboración propia a través de método de Brass, en base a los datos de XVI censo de población y vivienda, INE, Honduras, 2001.
Los resultados son mayores en el área rural que en el área urbana. Quizás esto se deba a que en el área rural las madres tienden a tener menor educación, embarazos en edades mayores y son más propensas a tener espacios más cortos entre embarazos. Como consecuencia, las muertes de niños están más concentradas en el área rural.

Los municipios seleccionados en Choluteca son los que presentan una mortalidad rural más alta 33 muertes por mil nacidos vivos; respecto a los municipios seleccionados en Valle de 26 muertes por mil nacidos vivos, los municipios de Francisco Morazán en situación intermedia encontrándose un diferencial por área de residencia de 9 muertes por mil nacidos vivos para Choluteca, 11 para Francisco Morazán, y 9 para Valle (tabla No. 2).

\section{VARIABLE EDUCACIÓN DE LA MADRE Y SU RELACIÓN CON LA MORTALIDAD EN LA NIÑEZ.}

En este apartado se muestran los resultados obtenidos de la estimación indirecta de la mortalidad en la niñez asociada al nivel educativo de la madre, medido por el número de años aprobado en la educación formal, situación esta que se describe por departamento y área de residencia.

Para analizar esta variable se categorizó así:

- Madres con ningún nivel educativo.

- Madres con nivel educativo primaria (en las que se agruparon las que han cursado años de alfabetización, preprimaria y primaria.).

- Madres con nivel de secundaria y más.

En el análisis por departamento los municipios seleccionados en Francisco Morazán presentan la tasa de mortalidad de 53 muertes por mil nacidos vivos en el nivel educativo ninguno, probablemente esto se deba al tamaño de la muestra bajo estudio, así como a los municipios involucrados en el mismo (tabla No. 3).

Se observa una notoria disminución en la medida que avanza el nivel educativo de la madre; tasas menores en el nivel primario y mucho más en el nivel secundario y más. El cambio es lineal con cada nivel educativo. Los resultados reflejan que existe un alto porcentaje de analfabetismo en las mujeres de estos municipios las que se han visto privadas de acceder a los servicios educativos, quizá porque tengan que incorporarse al proceso productivo a edades tempranas, o porque son marginadas o discriminadas por sus padres y no se encuentran en igualdad de condiciones con los varones para tener acceso a la educación.

Tasas de mortalidad en la niñez a partir del método de Brass variante Coale- Trussell según condiciones de la vivienda.

Esta sección se encamina principalmente a la descripción de la asociación entre la mortalidad en la niñez y algunas variables en lo que se refiere a las condiciones de la vivienda.

Tabla No. 3. Tasas de Mortalidad en la Niñez Estimadas a partir del Método de Brass variante Coale- Trussell según años de estudio de la madre por departamento.

$\begin{array}{lccc}\text { Departamento } & \text { Ninguno } & \text { Primaria } & \text { Secundaria y más } \\ \text { Choluteca } & 39 & 28 & 18 \\ \text { Francisco Morazán } & 53 & 24 & 15 \\ \text { Valle } & 37 & 24 & 13\end{array}$

Fuente: Elaboración propia a través de método de Brass, en base a los datos de XVI censo de población y vivienda, INE, Honduras, 2001. 
Con este propósito se seleccionaron los indicadores siguientes:

- Tipo de piso de la vivienda.

- Disposición de servicio sanitario en la vivienda.

- Instalación de tubería en la vivienda.

Tasas de mortalidad en la niñez según el tipo de piso de la vivienda por departamento.

Para hacer esta estimación se utilizó la pregunta que aparece en el censo de población del 2001, siempre a través del método indirecto.

El piso es una variable con mucha consistencia para la estimación de la mortalidad en la niñez, el piso de tierra es una condición que esta ligada a enfermedades diarreicas y parasitarias que al final conllevan a la muerte.

Para el manejo de esta variable se categorizó de la siguiente forma:

- Viviendas que poseen piso de tierra.

- Viviendas con otro tipo de piso (en el que se agruparon las que poseen piso de: plancha de cemento, ladrillo terrazo, ladrillo de barro cerámica y otro).

Como se observa en la tabla No. 4 la tasa de mortalidad en Choluteca y Francisco Morazán es 39 muertes por mil nacidos vivos en las madres residiendo en viviendas con piso de tierra y Valle con una tasa de 28 muertes por mil nacidos vivos; el diferencial entre las que tienen piso de tierra y otro tipo de piso es de 14 muertes por mil nacidos vivos para Choluteca 20 muertes por mil nacidos vivos para Francisco Morazán por tratarse de pequeñas poblaciones con características rurales, y 9 muertes por mil nacidos vivos para Valle.

El tipo de piso es indicativo del nivel económico de las familias. Los datos nos reflejan que, las condiciones de vida de un porcentaje considerable de estas familias viven en condiciones desfavorables para el desarrollo y crecimiento de sus hijos.

Tasas de mortalidad en la niñez estimadas a partir del método de Brass Variante Coale- Trussell (Modelo Oeste) según la disposición de servicio sanitario en la vivienda.

Así como el piso de la vivienda, el tipo de servicio sanitario en el hogar es un indicador de las condiciones de vida de una población

Tabla No. 4. Tasas de mortalidad en la niñez según el tipo de piso de la vivienda por departamento

\begin{tabular}{lcc} 
Departamento & Tierra & $\begin{array}{c}\text { Tipo de piso } \\
\text { Otro tipo de piso }\end{array}$ \\
\hline Choluteca & 39 & 25 \\
Francisco Morazán & 39 & 19 \\
Valle & 28 & 19
\end{tabular}

Fuente: Elaboración propia a través de método de Brass, en base a los datos de $\mathrm{XVI}$ censo de población y vivienda, INE, Honduras, 2001.

Tabla No. 5. Tasas de mortalidad en la niñez estimadas a partir del método de Brass Variante Coale- Trussell (Modelo Oeste) según la disposición de servicio sanitario en la vivienda

\begin{tabular}{lcc}
\multicolumn{1}{c}{ Departamento } & Con Sanitario & Sin Sanitario \\
Choluteca & 26 & 38 \\
Francisco Morazán & 24 & 34 \\
Valle & 20 & 30
\end{tabular}

Fuente: Elaboración propia a través de método de Brass, en base a los datos de $\mathrm{XVI}$ censo de población y vivienda, INE, Honduras, 2001. y cuando este no existe o es inadecuado se constituye en un foco de contaminación.

Para el análisis de esta variable las viviendas se clasifican en:

- Viviendas que no tienen sanitario.

- Viviendas con sanitario puede ser este de la red de alcantarillado público, letrina con pozo, sépticoletrina y otro.

Según esta variable en la tabla No. 5 se ve muy claro que las tasas más altas de mortalidad en la niñez se presentan en madres en cuya vivienda no hay servicio sanitario, 38 muertes por mil nacidos vivos para Francisco Morazán y 30 muertes por mil nacidos vivos para Valle, el diferencial en relación a las que si cuentan con servicio sanitario es de 12 muertes por mil nacidos vivos para Choluteca, 10 muertes por mil nacidos vivos para Francisco Morazán y de 10 muertes por mil nacidos vivos para Valle.

Los resultados muestran tasas de mortalidad superiores en las viviendas sin sanitario, lo que nos indica que un alto porcentaje de estas familias en un focos de infección sanitaria en las viviendas y constituyen una fuente de contaminación para el medio ambiente.

Tasas de mortalidad en la niñez estimada a partir del método de Brass variante Coale Trussell (modelo oeste) según la forma de abastecimiento de agua en la vivienda por departamento.

El agua es un recurso vital para la vida de los seres humanos y de la forma como se obtenga depende la calidad de vida de la misma, sabiendo anticipadamente que si se usa agua no tratada para el consumo humano se corre el riesgo de contraer enfermedades que conducen a la muerte.

Para el estudio de esta variable las viviendas se clasificaron así:

- viviendas con tubería del sistema público o privado

- viviendas que se abastecen de otro medio como pozo, río vertiente $\mathrm{u}$ otro.

Las tasas de mortalidad son mayores en donde el abastecimiento es de vertiente, río, quebrada u otro, siendo estas tasa de 38 por mil nacidos vivos para Francisco Morazán, 34 para Choluteca y 28 para Valle, observándose un diferencial en relación con las que se abastecen de agua de tubería pública o privada de 17 muertes por mil nacidos vivos para Francisco Morazán, 8 para Choluteca y 9 para Valle, siendo Francisco Morazán el departamento con las tasa más altas de mortalidad en la niñez en relación con esta variable (Tabla No. 6).

Tasas de mortalidad en la niñez estimadas a partir del método de Brass variante Coale Trussell (modelo oeste) según al forma de eliminación de la basura.

Como todas las otras variables la forma de eliminación de la basura tiene mucha importancia en relación con la mortalidad en la niñez. De la forma como se elimine la basura puede inferirse la calidad de vida de las familias.

Las madres que tiran la basura a la calle, además de contaminar

Tabla No. 6. Tasas de mortalidad en la niñez estimada a partir del método de Brass variante Coale Trussell (modelo oeste) según la forma de abastecimiento de agua en la vivienda por departamento

\begin{tabular}{lcc} 
Departamento & $\begin{array}{c}\text { Abastecimiento de sis- } \\
\text { tema público o privado }\end{array}$ & $\begin{array}{c}\text { Otra forma de } \\
\text { abastecimiento }\end{array}$ \\
\hline Choluteca & 26 & 34 \\
Francisco Morazán & 21 & 38 \\
Valle & 19 & 28
\end{tabular}

Fuente: Elaboración propia a través de método de Brass, en base a los datos de XVI censo de población y vivienda, INE, Honduras, 2001. 
el ambiente exponen a sus hijos a contraer enfermedades diarreicas a diferencia de las que la elimina de otra forma sus hijos tiene la posibilidad de una vida mas sana.

Para la estimación de la mortalidad en la niñez, en relación a la forma de eliminación de la basura se ha dividido esta variable en dos categorías:

- Las que tiran la basura a la calle.

- Las que la eliminan de otra forma donde se incluye las que la llevan al depósito, la queman o la entierran, paga a particulares, la recoge el tren de la basura y otros.

Los datos de la Tabla No. 7 muestran que el departamento que posee la mayor tasa de mortalidad en la niñez en relación a las madres que tiran la basura a la calle es para Choluteca 40 muertes por mil nacidos vivos, seguidos de Francisco Morazán con 38 muertes por mil nacidos vivos y Valle con 20 muertes por mil nacidos vivos al comparar estos datos con las madres que eliminan la basura de otra forma el diferencial es de 13 muertes por mil nacidos vivos para Choluteca, 12 muertes por mil nacidos vivos para Francisco Morazán manteniéndose la tabla estable para Valle. Probablemente esto se deba al tamaño de la muestra para este departamento.

Tabla No. 7. Tasas de mortalidad en la niñez estimadas a partir del método de Brass variante Coale Trussell (modelo oeste) según al forma de eliminación de la basura.

\begin{tabular}{lcc}
\hline Departamento & $\begin{array}{c}\text { Tiran la basura a } \\
\text { la calle }\end{array}$ & $\begin{array}{c}\text { Otra forma } \\
\text { de eliminación }\end{array}$ \\
\hline Choluteca & 40 & 27 \\
Francisco Morazán & 38 & 26 \\
Valle & 20 & 20
\end{tabular}

Fuente: Elaboración propia a través de método de Brass, en base a los datos de XVI censo de población y vivienda, INE, Honduras, 2001.

\section{REFLEXIONES FINALES}

- En cuanto al aspecto metodológico para el análisis de la mortalidad en la niñez se hizo de manera indirecta, en base a la información sobre hijos nacidos vivos e hijos sobrevivientes por edad de la madre, tomando como base de datos el censo del 2001. Como bien la aclara W. Brass el método no se recomienda se utilice para el análisis de pequeñas poblaciones, entonces para efectos del presente estudio se agruparon los municipios en su respectivo departamento.

- Se puede afirmar que existe cierta evidencia de que la información sobre hijos nacidos vivos e hijos sobrevivientes recopilada en el censo del 2001, permite analizar la tendencia de la mortalidad en la niñez ya sea a nivel general o por características sociales y económicas.

- Al hacer el análisis por área de residencia los datos nos muestran que es en el área rural donde se encuentran las tasas más altas de mortalidad en la niñez en relación con el área urbana, probablemente se deba a que las mujeres en esta área contraen matrimonio a edades tempranas, a que el espacio ínter genésico sea muy corto, o que el acceso a los servicios de salud es limitado.

- Con respecto a los diferenciales de mortalidad en la niñez según nivel educativo de la madre, se concluye que la mortalidad en la niñez alcanza tasas más altas cuando la madre no posee ningún nivel educativo en relación al nivel primario, secundaria y más, se observa una tendencia generalizada con relación al área de residencia, en los tres departamentos, aunque con menores logros en los municipios de Francisco Morazán. Los resultados obtenidos vienen a validar la hipótesis planteada al inicio del presente estudio. Se considera que las mujeres más educadas son más autosuficientes, más cuidadosas en la preparación de los alimentos y cuidado de sus hijos, demandan más tratamiento para sus pequeños y son más capaces de seguir instrucciones para el tratamiento de la enfermedad.

- En cuanto a la mortalidad en la niñez y las condiciones de la vivienda, indicador de las condiciones socioeconómicas de las familias, se concluye que las tasas de mortalidad en la niñez son más altas en las madres residiendo en viviendas con piso de tierra en relación con las madres residiendo en viviendas que poseen otro tipo de piso, tendencia general en los departamentos bajo estudio y área de residencia, con mayores logros en los municipios de Valle. Los resultados indican que estas familias están más expuestas a contraer enfermedades diarreicas y parasitarias.

- En relación a la disponibilidad de servicio sanitario y mortalidad en la niñez, se encuentran tasas más bajas de mortalidad en la niñez en las viviendas que disponen de servicio sanitario, y tasas más altas de mortalidad en donde las viviendas no poseen servicio sanitario. Lo que hace suponer que estas familias al depositar sus heces fecales al aire libre se exponen más a la contaminación, por tanto sus hijos están más propensos a enfermarse.

- Otro condicionante en relación a la mortalidad en la niñez, es la forma de abastecimiento de agua, encontrándose tasas más altas de mortalidad en la niñez en las viviendas que no poseen tubería en relación a las que si poseen, se observó además una tendencia general por departamento y área de residencia, con mayores logros para los municipios de Valle y menores logros para los municipios de Francisco Morazán.

- Existen diferencias claras en relación a la mortalidad en la niñez y la forma de eliminación de la basura. Se encontraron valores más bajos cuando las madres eliminan la basura de otras formas y valores más altos cuando tiran la basura a la calle exponiendo a sus hijos a contraer enfermedades diarreicas y parasitarias. Los resultados mostraron que los municipios de Choluteca presentan menores logros y los municipios de Valle con mayores logros. Los resultados obtenidos entre la relación que existe entre mortalidad en la niñez y condiciones de la vivienda vienen a validar la hipótesis planteada al inicio del documento.

- Los resultados encontrados en el estudio, establecen que la asociación entre mortalidad en la niñez y las condiciones de la vivienda es menos marcada en comparación con la relación entre mortalidad en la niñez y la educación de la madre.

- La mortalidad en la niñez es un indicador del desarrollo socioeconómico alcanzado por una población. En los municipios de los tres departamentos bajo estudio se concluye que, Francisco Morazán es el departamento con tasas más altas de mortalidad en la niñez en casi todas las variables bajo estudio por lo que se asume que las condiciones de vida de sus habitantes son precarias le sigue el departamento de Choluteca y en mejor situación el departamento de Valle.

Los resultados encontrados en la investigación mostraron cuáles son los determinantes que más afectan la mortalidad en niños y niñas menores de cinco años (nivel educativo de la madre, condiciones de la vivienda, área de residencia), por lo que se espera que estos, sirvan a las autoridades de los municipios estudiados en la toma de decisiones y en la aplicación de políticas públicas 
relacionadas con la educación, salud e infraestructura, que venga a mejorar la situación económica y social en la que viven las familias de estas comunidades, para que les permita tener una vida de calidad.

\section{BIBLIOGRAFÌA}

- Albizu-Campos Espiñeira, 2002, "Mortalidad y Supervivencia en Cuba en los 90", Universidad de la Habana, Centro de estudios Demográficos. LA Habana, Cuba. - Banguero, Harold, 2000, "La Mortalidad", Lectura № 8, UNAH- PLATS, Tegucigalpa, Honduras.

- Banguero, Harold, 2000, "La Transición Demográfica en Honduras", UNAHPLATS, Tegucigalpa, Honduras.

- Centro Latinoamericano de Demografía (CELADE), 1988 Recuperación de DATos Censales para Áreas Pequeñas por Microcomputador, (REDATAM) Versión 3.0, Santiago de Chile.

- Centro Latinoamericano de Demografía (CELADE), 1999, Boletín № 62, Santiago de Chile.

- Centro Latinoamericano de Demografía (CELADE), Paquete de Análisis Demo- gráfico (PANDEM), 1989, Versión № 2.0, Santiago de Chile.

- Encuesta Nacional de Epidemiologia y Salud Familiar, (ENESF), 2001, Tegucigalpa, Honduras.

- Facultad Latino Americana de Ciencias Sociales, (FLACSO), "Centro América En Cifras 1980-2000", 2002, 1² Edición, San José, Costa Rica. - García, Molina, Carlos y Hernández, Héctor, "Mortalidad, Salud y Discurso Demográfico", 1996, $1^{\circ}$ Edición, Cuernavaca, México.

- Hakkert, Ralp, (sin fecha) "La Población de Honduras una Síntesis", (Documento Mimeografiado), Tegucigalpa, Honduras.

- HTTP// w.w.w. sise. Gov.ec/fichas/salu05v8htm.

- Naciones Unidas ,Informe Mundial Sobre El Desarrollo, 2003

- Naciones Unidas, Técnicas Indirectas de Estimación Indirecta, Manual X, 1983. - OPS, Estadistica de Salud en las Américas, "La Salud en la niñez Temprana, Mortalidad Registrada, 1988

- Rodríguez Leticia, "Diferenciales Geográficos y Socioeconómicos de la Mortalidad Infantil en Honduras 1975-1985", 1992, Tegucigalpa, Honduras.

- Secretaría de Salud, "Morbilidad y Mortalidad Neonatal en el Área Rural", 1986, Tegucigalpa, Honduras.

- UNICEF, Estado Nacional de la Infancia, 1999, Tegucigalpa, Honduras. - Welti, Carlos, "Demografía I", 1997,1 Edición, México, México. 